TITLE:

\title{
A novel strictly NADPH-dependent Pichia stipitis xylose reductase constructed by site-directed mutagenesis.
}

\section{AUTHOR(S):}

Khattab, Sadat Mohammad Rezq; Watanabe, Seiya; Saimura, Masayuki; Kodaki, Tsutomu

\section{CITATION:}

Khattab, Sadat Mohammad Rezq ... [et al]. A novel strictly NADPH-dependent Pichia stipitis xylose reductase constructed by site-directed mutagenesis.. Biochemical and biophysical research communications 2011, 404(2): 634-637

\section{ISSUE DATE:}

2011-01-14

URL:

http://hdl.handle.net/2433/134797

\section{RIGHT:}

(C) 2010 Elsevier Inc.; This is not the published version. Please cite only the published version.; この論文は出版社版でありません。引用の際に は出版社版をご確認ご利用ください。 
A Novel Strictly NADPH-Dependent Pichia stipitis Xylose Reductase Constructed by

Site-directed Mutagenesis

Sadat Mohammad Rezq Khattab ${ }^{1,2}$, Seiya Watanabe ${ }^{1}$, Masayuki Saimura ${ }^{1}$,

${ }^{1}$ Institute of Advanced Energy, Kyoto University, Gokasho, Uji, Kyoto 611-0011, Japan

${ }^{2}$ Faculty of Science, Al-Azhar University, Assiut Branch 71524, Egypt

Abstract

Xylose reductase (XR) and xylitol dehydrogenase (XDH) are the key enzymes for

10 xylose fermentation and have been widely used for construction of a recombinant xylose

11 fermenting yeast. The effective recycling of cofactors between XR and XDH has been

12 thought to be important to achieve effective xylose fermentation. Efforts to alter the 13 coenzyme specificity of XR and HDX by site-directed mutagenesis have been widely

14 made for improvement of efficiency of xylose fermentation. We previously succeeded by

15 protein engineering to improve ethanol production by reversing XDH dependency from

$16 \mathrm{NAD}^{+}$to $\mathrm{NADP}^{+}$. In this study, we applied protein engineering to construct a novel

17 strictly NADPH dependent XR from Pichia stipitis by site-directed mutagenesis, in order

18 to recycle NADPH between XR and XDH effectively. One double mutant, E223A/S271A

19 showing strict NADPH dependency with $106 \%$ activity of wild-type was generated. A

20 second double mutant, E223D/S271A, showed a 1.27-fold increased activity compared to

21 the wild-type XR with NADPH and almost negligible activity with NADH.

22

23 Keywords: Coenzyme specificity, Xylose reductase, Site-directed mutagenesis 24 
$25 *$ Corresponding author. Mailing address: Institute of Advanced Energy, Kyoto University,

26 Gokasyo, Uji 611-0011, Japan Phone: 81-774-38-3510. Fax: 81-774-38-3524.

27 E-mail: kodaki@iae.kyoto-u.ac.jp

\section{1. Introduction}

Xylose is the second most abundant pentose sugar constituting the lignocellulosic

31 renewable biomass after glucose, and its complete fermentation is economically valuable

32 for producing biofuel from lignocellulosic biomass [6]. Recombinant S. cerevisiae can

33 ferment xylose through a fungal pathway involving two heterologous oxidoreductase

34 genes. In this pathway, Pichia stipitis xylose reductase (PsXR) (XR; EC 1.1.1.21) [18],

35 which prefers NADPH, reduces xylose to xylitol followed by $P$. stipitis xylitol

36 dehydrogenase (PsXDH), which exclusively requires $\mathrm{NAD}^{+}(\mathrm{XDH}$; EC 1.1.1.9) [17],

37 oxidizes xylitol into xylulose. S. cerevisiae xylulokinase (XK) (EC 2.7.1.17) naturally

38 phosphorylates xylulose to xylulose-5-phosphate, which is then metabolized by the

39 glycolytic pathway via the pentose phosphate pathway [7]. XK overexpression improves

40 the efficiency of xylose fermentation [4, 5, 15]. Although this fungal pathway is highly

41 expressed in S. cerevisiae, the efficiency of ethanol production is somewhat obstructed by

42 the unfavorable accumulation of xylitol due to the imbalance of coenzyme specificities

43 between $\mathrm{XR}$ and $\mathrm{XDH}[6]$.

44 Xylose reductase is a member of the aldo-keto reductase (AKR) superfamily 45 which is made up of 14 different families and approximately 120 members with a 46 majority of dual cofactor type enzymes [8]. Candida tenuis XR (CtXR) is one of these 47 enzymes. Its crystal structure has been determined at different levels of resolution and its 48 binding sites with $\mathrm{NAD}(\mathrm{P}) \mathrm{H}$ were also determined $[10,12,25]$. Although only little 
49 structural information of PsXR is available, it has about $76 \%$ homology with the CtXR.

50 This high percentage of similarity should provide some clues for manipulation of PsXR

$51[13]$.

Protein engineering has been widely used to alter the coenzyme specificity of XR

53 and $\mathrm{XDH}$. Since PsXDH accepts only $\mathrm{NAD}^{+}$, many researchers reversed the preference

54 of $\mathrm{XR}$ to $\mathrm{NADH}$ in order to achieve $\mathrm{NAD}^{+} / \mathrm{NADH}$ cofactor recycling $[1,13,16,20]$. On

55 the other hand, we have been working on converting cofactor usage of XDH to NADP ${ }^{+}$

56 from $\mathrm{NAD}^{+}[21]$. We previously succeeded to improve the fermentation process and

57 ethanol production by using these $\mathrm{XDH}$ mutants [23]. In this study, site-directed

58 mutagenesis of PsXR was performed to construct a strictly NADPH-dependent XR,

59 expecting decreasing or preventing xylitol accumulation and subsequently improving

60 ethanol production.

61

\section{2. Materials and Methods}

63 2.1 Cloning of the $P$. stipitis Xylose reductase gene and Site-directed mutagenesis

A plasmid, named pHis (WT) harboring the His-tagged wild-type (WT) PsXR

65 gene was constructed as described previously [20]. All XR mutations were introduced by

66 site-directed mutagenesis, using the single round PCR method with PfuTurbo DNA

67 polymerase (Stratagene) and the PCR Thermal Cycler PERSONAL (TaKaRa, Otsu,

68 Japan). The codons used for mutations introduced in this study were as follows: E223A

69 (GAA $\rightarrow$ GCA), E223D (GAA $\rightarrow$ GAC), and S271A (TCC $\rightarrow$ GCC). The PCR products

70 were subjected to DpnI restriction enzyme treatment in order to digest the parent DNA

71 strands to prevent transformation of the template plasmid. Only nicked circular

72 mutagenic strands were transformed into Escherichia coli DH5a. Electroporation method 
73 was used to transform plasmids and the mutations were confirmed by DNA sequencing

74 using Applied Biosystems 3031 genetic analyzer and ABI Prism ${ }^{\circledR}$ Big Dye $^{\circledR}$ Terminator

75 v3.1 Cycle Sequencing kit.

76

772.2 Overexpression and purification of (His) $)_{6}$-tagged enzymes

$P$. stipitis xylose reductase wild-type and mutated genes were expressed in E. coli

79 DH5 $\alpha$ and purified as described previously [20]. Purified enzymes were confirmed on

$8010 \%$ acrylamide SDS-PAGE. Protein concentrations were determined using the Bio

81 RAD Quick Start Bradford 1x Dye Reagent (Bio-Rad Laboratories, CA, USA) by

82 measuring the absorbance at $595 \mathrm{~nm}$ with $\gamma$-globin as a standard.

$84 \quad 2.3$ Enzyme assays and Kinetic parameters

Enzyme activities were measured spectrophotometrically as described previously

86 [20] with modification in xylose concentration to $400 \mathrm{mM}$. The kinetic parameters were

87 calculated by Line Weaver-Burk plots.

\section{3. Result and Discussion}

$90 \quad 3.1$ Speculation and prediction of $\mathrm{NAD}(\mathrm{P}) \mathrm{H}$ binding sites

91 Crystallographic analyses of many AKRs have revealed that they share a common

$92(\alpha / \beta)_{8}$ barrel fold, with a highly conserved coenzyme binding pocket at the C-terminus.

$9390.9 \%$ of the residues are located in the core area and $9.1 \%$ are in the allowed regions

94 [11]. The nicotinamide ring of $\mathrm{NAD}(\mathrm{P}) \mathrm{H}$ is resides in the core of the barrel. Residues

$95 \mathrm{Glu}^{227}$ and $\mathrm{Asn}^{276}$ in CtXR, which equal to Glu ${ }^{223}$ and $\mathrm{Asn}^{272}$ in PsXR, primarily mediate

96 the interactions with the adenosine ribose 2'- and 3'-hydroxy groups. As shown in Fig. 1, 
$97 \mathrm{Glu}^{223}$ represents the essential part NADH binding where contacts by bidentate hydrogen

98 bond with both of the hydroxy groups. Similar interactions have been seen in many other

99 NADH-binding protein structures [2, 3]. However, The structurally equivalent residues

$100 \mathrm{Asp}^{216}$ and $\mathrm{Val}^{264}$ in aldose reductase in human (AR) are unable to fulfill the equivalent

101 roles, $\mathrm{Asp}^{216}$ is required for high affinity binding of NADPH by forming two salt linkages

102 with Lys $^{21}$ and Lys ${ }^{262}$ and fastening the loop over the co-substrate [24].

$103 \mathrm{Glu}^{227}$ and $\mathrm{Lys}^{274}$ in CtXR makes water-mediated interactions each other and with

104 the 3'-hydroxy group in the case of $\mathrm{NADP}^{+}$-bound structures. In the absence of a 105 negatively charged phosphate, $\mathrm{Glu}^{227}$ side chain is able to rotate into a favourable 106 conformation to accept a $2.64 \AA$ hydrogen bond contact with the 2'-hydroxy group and a

$1072.65 \AA$ A hydrogen bond with the 3'-hydroxy group when $\mathrm{NAD}^{+}$is bound. The root mean 108 square deviations of the $\mathrm{C} \alpha$ values between $\mathrm{NAD}^{+}$- and $\mathrm{NADP}^{+}$-bound models was 109 calculated in CtXR. The largest conformational change is seen in residues 274-280, 110 which corresponding to 270-276 in PsXR, and then residues 225-229, which 111 corresponding to 221-225 in PsXR, a short helical region that appears at the end of $\beta 7$.

112 The largest main-chain shift is seen in $\operatorname{Ser}^{275}$, which corresponding to $\operatorname{Ser}^{271}$ in PsXR, 113 moves $2.0 \AA$ in response to the miss contact of the phosphate group of NADPH [10].

114 Furthermore, Glu ${ }^{223}$ of PsXR was subjected to a mutation trial and the result revealed that 115 alteration of this site might further inhibit NADH binding [13]. In addition, from the 3D 116 structure model of PsXR, it was reported that $\mathrm{Glu}^{223}$ and $\mathrm{Phe}^{236}$ can form 3 and 2 117 hydrogen bonds with $\mathrm{NAD}^{+}$, respectively [19].

118 Considering the property as described above, the mutations were designed based 119 on sequence alignment of some strictly NADPH dependent analogous enzymes in the 120 AKR family, such as AR, as shown in table1, where glutamic acid 223 was substituted by 
121 aspartic acid. Both glutamic and aspartic acid are acidic side chain and fully ionized at

122 neutral $\mathrm{pH}$ and able to engage in hydrogen bonds, which is a necessary component for a

123 high affinity xylose binding site [9]. Alanine is a nonpolar side chain that does not bind

124 or give off protons, or participate in hydrogen or ionic bonds. Alanine can be worked as

125 oily or lipid-like that promotes hydrophobic interactions. Accordingly, we apply aspartic

126 acid and alanine to mutation trials instead of PsXR glutamic acid 223.

3.2 Strictly NADPH dependency on Glu ${ }^{223}$ mutants

We applied $\mathrm{Glu}^{223}$ residue for mutation trails in order to delete NADH dependency.

130 Although this residue is also shared in NADPH binding, some reports reveal that it

131 contributes more to the affinity of $\mathrm{NADH}$, where it plays a role in the binding site by

132 binding two hydrogen bonds with $2^{\prime}$ and 3 ' hydroxy groups of the adenosine ribose. In

133 addition to changes in hydrogen-bonding of the adenosine, the ribose unmistakably

134 adopts the 3'-endo conformation rather than the 2'-endo conformation seen in the NADP ${ }^{+}$-

135 bound form [10]. The enzyme activities with NADH were calculated after introduction of

$136 \mathrm{Glu}^{223}$ residue mutations (Fig. 2). No activity was detected for E223A with NADH while

137 E223D showed only $17 \%$ of the activity of WT. In addition, catalytic efficiency was

138 decreased to $3.7 \%$ of WT. Their activities with NADPH showed $52 \%$ and $44 \%$ of WT,

139 respectively. The catalytic efficiencies of E223A and E223D were $26 \%$ and $15 \%$ of WT

140 respectively. Although, these ratios were low compared with WT, E223D showed 2.54

141 and 3.9 fold improvement in NADPH/NADH ratio and $k_{c a t} / K_{m}$ respectively. E223A is a

142 completely NADPH dependent mutant, probably due to the change of 3'-endo ribose

143 conformation and miss contact of bidentate hydrogen bonds which was conserved in

$144 \mathrm{NAD}^{+}$binding sites in most members of dual cofactor in AKR family. 
1463.3 Improvement of enzyme activities with double mutants

147 We previously reported that the mutation of $\mathrm{Lys}^{270}$ and $\mathrm{Arg}^{276}$ in PsXR improve 148 NADH preference [20], while S271A increased the preference for NADPH [22]. The 149 second rounds of mutations were done based on this data. Accordingly, combination of 150 S271A with Glu ${ }^{223}$ mutants was expected to increase the activity of XR with NADPH. As 151 shown in Fig. 1, S271A mutant showed improved NADPH preference, where the 152 activities with NADPH and NADH were $125 \%$ and $85 \%$ compared to WT respectively. 153 These data encouraged us to perform further investigations by combining S271A and $154 \mathrm{Glu}^{223}$ mutants. A combination of site-directed mutations of the residues $\mathrm{Glu}^{223}$ and 155 S271A produced unique and unprecedented results. The double mutants E223A/S271A 156 (AA) and E223D/S271A (DA) showed improvement in the activities with NADPH 157 compared to single Glu ${ }^{223}$ mutants. As shown in Fig. 2, the activity of the double mutant 158 AA with NADPH was $106 \%$ compared to WT. As shown in Table 2 , the $k_{\text {cat }}$ of WT and 159 AA were 622 and $657 \mathrm{~min}^{-1}$, respectively; their $K_{\mathrm{m}}$ for xylose were 97.1 and $226 \mathrm{mM}$, 160 respectively; and their catalytic efficiencies were 38.6 and $32.4 \mu \mathrm{M}^{-1} / \mathrm{min}^{-1}$, respectively. 161 On the other hand, the activity of DA showed $15 \%$ WT with NADH (Fig. 1) in addition 162 to $K_{\mathrm{m}}$ was increased 12.8-fold and $k_{\text {cat }}$ decreased 3-fold (Table 2). As shown in Fig. 2, the 163 activity of DA with NADPH was increased 1.27 -fold compared to WT; $k_{\text {cat }}$ also increased 164 1.18-fold compared to WT, while catalytic efficiency was decreased to $93 \%$ of WT and $165 K_{\mathrm{m}}$ increased 1.26-fold compared to WT (see table 2). Thus we succeeded to construct a 166 novel strictly NADPH-dependent PsXR by combining the mutation at Glu ${ }^{223}$ and $\mathrm{Ser}^{271}$ 167 residues. 
168 We previously succeeded in improving xylose fermentation and ethanol production

169 by combining PsXR WT with the mutated PsXDH which accepts only $\mathrm{NADP}^{+}$(i.e., 170 quadruple ARSdR mutant) [23], and overexpression of XK [14, 15]. It may provide

171 further clues for understanding of importance of coenzyme specificities of XR and XDH

172 using the strictly NADPH-dependent PsXR of this study together with the strictly

$173 \mathrm{NADP}^{+}$-dependent PsXDH [21]. It could possibly give more efficient xylose

174 fermentation by an effective recycling of coenzymes of NADPH between XR and XDH.

176 Acknowledgements

177 This work was supported by the New Energy and Industrial Technology

178 Development Organization (NEDO), Japan. It was also supported by the Global Center of

179 Excellence (GCOE) program for the "Energy Science in the Age of Global Warming," a 180 Grant-in-Aid for Scientific Research from the Ministry of Education, Science, Sports, and 181 Culture, Japan.

183 References

184 [1] O. Bengtsson, B. Hahn-Hagerdal, M.F. Gorwa-Grauslund, Xylose reductase from Pichia stipitis with altered coenzyme preference improves ethanolic xylose fermentation by recombinant Saccharomyces cerevisiae, Biotechnol. Biofuels 2 (2009) 9 .

189 [2] O. Carugo, P. Argos, NADP-dependent enzymes. 1. Conserved stereochemistry of 190 cofactor binding, Proteins Struct. Funct. and Genet. 28 (1997) 10-28. 
192 [3] O. Carugo, P. Argos, NADP-dependent enzymes. 2. Evolution of the mono- and dinucleotide binding domains, Proteins Struct. Funct. and Genet. 28 (1997) 29-40.

[4] A. Eliasson, C. Christensson, C.F. Wahlbom, B. Hahn-Hagerdal, Anaerobic xylose fermentation by recombinant Saccharomyces cerevisiae carrying XYL1, XYL2, and XKS1 in mineral medium chemostat cultures, Appl. Environ. Microbiol. 66 (2000) 3381-3386.

[5] A. Eliasson, J.H.S. Hofmeyr, S. Pedler, B. Hahn-Hagerdal, The xylose reductase/xylitol dehydrogenase/xylulokinase ratio affects product formation in recombinant xylose-utilising Saccharomyces cerevisiae, Enzyme and Microbial. Technology 29 (2001) 288-297.

[6] T.W. Jeffries, Y.S. Jin, Metabolic engineering for improved fermentation of pentoses by yeasts, Appl. Microbiol. Biotechnol. 63 (2004) 495-509.

[7] H. Jeppsson, S. Yu, B. Hahn-Hagerdal, Xylulose and glucose fermentation by Saccharomyces cerevisiae in chemostat culture, Appl. Environ. Microbiol. 62 (1996) 1705-1709.

212 [8] J.M. Jez, T.M. Penning, The aldo-keto reductase (AKR) superfamily: an update, Chem. Biol. Interact. 130-132 (2001) 499-525.

215 [9] K.L. Kavanagh, M. Klimacek, B. Nidetzky, D.K. Wilson, The structure of apo and 
holo forms of xylose reductase, a dimeric aldo-keto reductase from Candida tenuis, Biochemistry 41 (2002) 8785-8795.

[10] K.L. Kavanagh, M. Klimacek, B. Nidetzky, D.K. Wilson, Structure of xylose reductase bound to $\mathrm{NAD}^{+}$and the basis for single and dual co-substrate specificity in family 2 aldo-keto reductases, Biochem. J. 373 (2003) 319-326.

[11] R.A. Laskowski, M.W. Macarthur, D.S. Moss, J.M. Thornton, Procheck - a Program to Check the Stereochemical Quality of Protein Structures, Journal of Applied Crystallography 26 (1993) 283-291.

[12] S. Leitgeb, B. Petschacher, D.K. Wilson, B. Nidetzky, Fine tuning of coenzyme specificity in family 2 aldo-keto reductases revealed by crystal structures of the Lys-274 $\rightarrow$ Arg mutant of Candida tenuis xylose reductase (AKR2B5) bound to $\mathrm{NAD}^{+}$and NADP ${ }^{+}$, Febs Letters 579 (2005) 763-767.

[13] L. Liang, J. Zhang, Z. Lin, Altering coenzyme specificity of Pichia stipitis xylose reductase by the semi-rational approach CASTing, Microb. Cell Fact. 6 (2007) 36.

[14] A. Matsushika, H. Inoue, S. Watanabe, T. Kodaki, K. Makino, S. Sawayama, Efficient bioethanol production by a recombinant flocculent Saccharomyces cerevisiae strain with a genome-integrated $\mathrm{NADP}^{+}$-dependent xylitol dehydrogenase gene, Appl. Environ. Microbiol. 75 (2009) 3818-3822. 
240 [15] A. Matsushika, S. Watanabe, T. Kodaki, K. Makino, S. Sawayama, Bioethanol production from xylose by recombinant Saccharomyces cerevisiae expressing xylose reductase, $\mathrm{NADP}^{+}$-dependent xylitol dehydrogenase, and xylulokinase, J. Biosci. Bioeng. 105 (2008) 296-299.

[16] B. Petschacher, B. Nidetzky, Altering the coenzyme preference of xylose reductase to favor utilization of $\mathrm{NADH}$ enhances ethanol yield from xylose in a metabolically engineered strain of Saccharomyces cerevisiae, Microb. Cell Fact. 7 (2008) 9 .

[17] M. Rizzi, K. Harwart, P. Erlemann, N.A. Buithanh, H. Dellweg, Purification and Properties of the NAD ${ }^{+}$-Xylitol-Dehydrogenase from the Yeast Pichia stipitis,

[18] C. Verduyn, R. Van Kleef, J. Frank, H. Schreuder, J.P. Van Dijken, W.A. Scheffers, Properties of the $\mathrm{NAD}(\mathrm{P}) \mathrm{H}-$ dependent xylose reductase from the xylosefermenting yeast Pichia stipitis, Biochem. J. 226 (1985) 669-677.

[19] J.F. Wang, D.Q. Wei, Y. Lin, Y.H. Wang, H.L. Du, Y.X. Li, K.C. Chou, Insights

263 [20] S. Watanabe, A. Abu Saleh, S.P. Pack, N. Annaluru, T. Kodaki, K. Makino, Ethanol 
production from xylose by recombinant Saccharomyces cerevisiae expressing protein-engineered NADH-preferring xylose reductase from Pichia stipitis, Microbiology 153 (2007) 3044-3054.

[21] S. Watanabe, T. Kodaki, K. Makino, Complete reversal of coenzyme specificity of xylitol dehydrogenase and increase of thermostability by the introduction of structural zinc, J. Biol. Chem. 280 (2005) 10340-10349.

[22] S. Watanabe, S.P. Pack, A.A. Saleh, N. Annaluru, T. Kodaki, K. Makino, The positive effect of the decreased NADPH-preferring activity of xylose reductase from Pichia stipitis on ethanol production using xylose-fermenting recombinant Saccharomyces cerevisiae, Biosci. Biotechnol. Biochem. 71 (2007) 1365-1369.

[23] S. Watanabe, A.A. Saleh, S.P. Pack, N. Annaluru, T. Kodaki, K. Makino, Ethanol production from xylose by recombinant Saccharomyces cerevisiae expressing protein engineered NADP ${ }^{+}$-dependent xylitol dehydrogenase, J. Biotechnol. 130 (2007) 316-319.

[24] D.K. Wilson, K.M. Bohren, K.H. Gabbay, F.A. Quiocho, An unlikely sugar substrate site in the $1.65 \mathrm{~A}$ structure of the human aldose reductase holoenzyme implicated in diabetic complications, Science 257 (1992) 81-84.

[25] D.K. Wilson, K.L. Kavanagh, M. Klimacek, B. Nidetzky, The xylose reductase (AKR2B5) structure: homology and divergence from other aldo-keto reductases 

$515-521$. 
Table1

The mutation designs of the PsXR enzyme

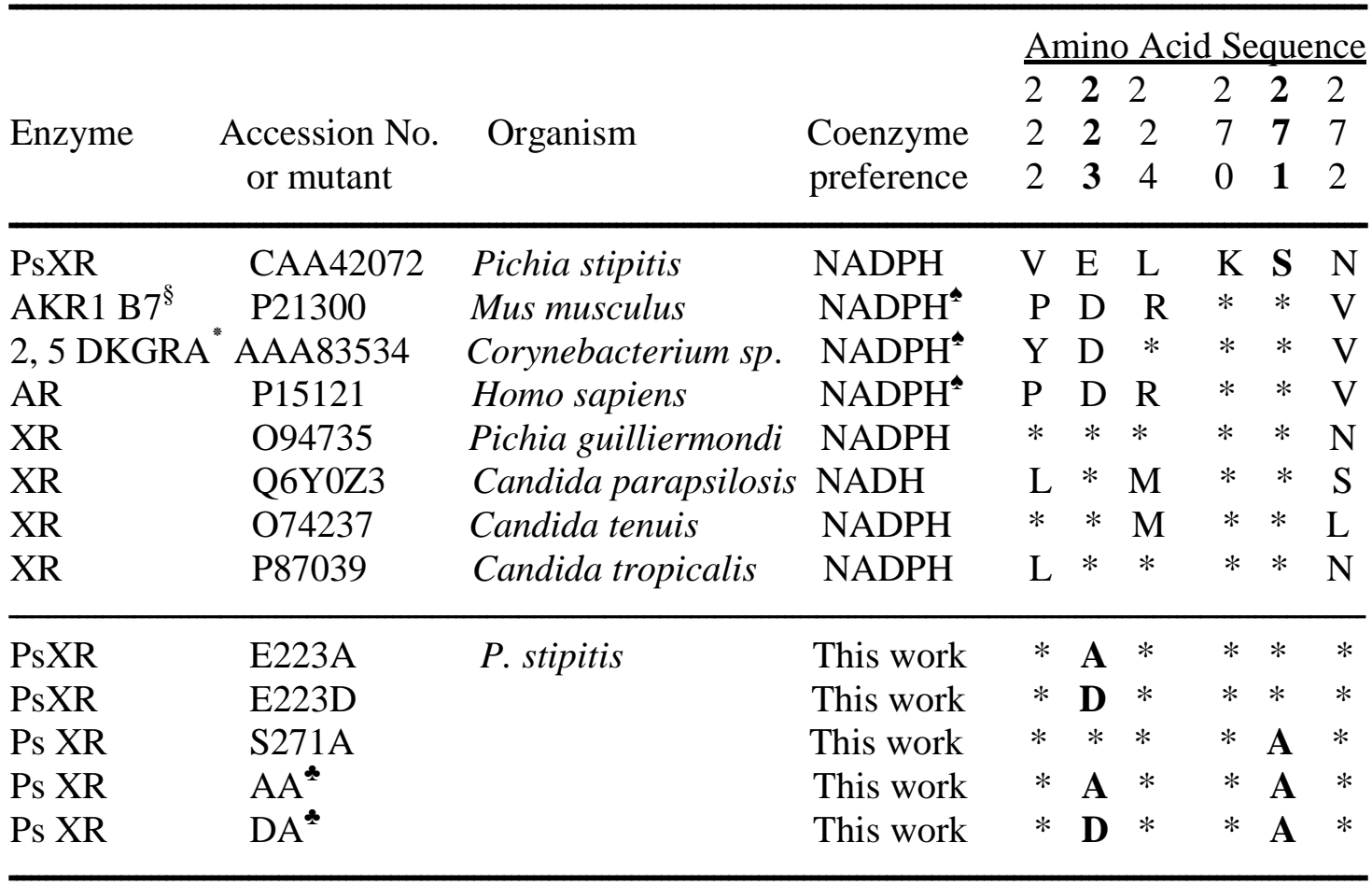

Bold letters represent target mutation sites

*The same amino acids as PsXR WT

* Double mutant E223A/S271A (AA) and E223D/S271A (DA)

* Strict NADPH dependent enzyme § Aldo-keto reductase family 1, memberB7

* 2,5-Diketo-D-gluconic acid reductase 
Table 2

Kinetic parameters of wild-type and xylose reductase mutants for NADPH- and NADH-dependent reactions

\begin{tabular}{|c|c|c|c|c|c|c|c|c|}
\hline \multirow[b]{3}{*}{ Enzymes } & \multirow{2}{*}{\multicolumn{4}{|c|}{ Kinetic paral }} & & & & \\
\hline & & & & & \multicolumn{4}{|c|}{ NADH } \\
\hline & $\begin{array}{c}K_{\mathrm{m}} \text { xylose }^{\mathrm{a}} \\
{[\mathrm{mM}]}\end{array}$ & $\begin{array}{l}K_{\mathrm{m}}^{\mathrm{b}} \\
{[\mu \mathrm{M}]}\end{array}$ & $\begin{array}{l}k_{\mathrm{cat}}^{\mathrm{b}} \\
{\left[\mathrm{Min}^{-1}\right]}\end{array}$ & $\begin{array}{l}k_{\mathrm{cat}} / K_{\mathrm{m}} \\
{\left[\mu \mathrm{M}^{-1} / \mathrm{min}^{-1}\right]}\end{array}$ & $\begin{array}{c}K_{\mathrm{m}} \mathrm{xylose}^{\mathrm{a}} \\
{[\mathrm{mM}]}\end{array}$ & $\begin{array}{l}K_{\mathrm{m}}^{\mathrm{b}} \\
{[\mu \mathrm{M}]}\end{array}$ & $\begin{array}{c}k_{\mathrm{cat}}^{\mathrm{b}} \\
{\left[\mathrm{min}^{-1}\right]}\end{array}$ & $\begin{array}{l}k_{\text {cat }} / K_{\mathrm{m}} \\
{\left[\mu \mathrm{M}^{-1} / \mathrm{min}^{-1}\right]}\end{array}$ \\
\hline XRWT & $97.1 \pm 4.8$ & $16.2 \pm 1.4$ & $622 \pm 22$ & $38.6 \pm 2.9$ & $170 \pm 23$ & $30.6 \pm 1.0$ & $449 \pm 22$ & $14.7 \pm 1.4$ \\
\hline S271A & $70.6 \pm 8.7$ & $30.1 \pm 3.7$ & $874 \pm 50$ & $29.0 \pm 0.3$ & $180 \pm 12$ & $53.3 \pm 4.4$ & $480 \pm 42$ & $9.00 \pm 0.40$ \\
\hline E223A & $29.8 \pm 4.7$ & $35.2 \pm 3.7$ & $349 \pm 29$ & $9.94 \pm 0.44$ & $\mathrm{ND}^{\mathrm{d}}$ & ND & ND & ND \\
\hline E223D & $114 \pm 13$ & $55.4 \pm 7.1$ & $314 \pm 42$ & $5.65 \pm 0.32$ & $376 \pm 32$ & $305 \pm 11$ & $169 \pm 52$ & $0.55 \pm 0.15$ \\
\hline $\mathrm{AA}^{\mathrm{c}}$ & $226 \pm 22$ & $17.5 \pm 0.7$ & $567 \pm 73$ & $32.4 \pm 2.7$ & ND & ND & ND & ND \\
\hline $\mathrm{DA}^{\mathrm{c}}$ & $108 \pm 8$ & $20.4 \pm 0.4$ & $733 \pm 14$ & $36.0 \pm 0.3$ & $353 \pm 1$ & $391 \pm 46$ & $156 \pm 29$ & $0.44 \pm 0.10$ \\
\hline
\end{tabular}

${ }^{a}$ Six different concentrations of xylose between 67 and $200 \mathrm{mM}$ were used and NAD(P)H concentration was $150 \mu \mathrm{M}$.

${ }^{\mathrm{b}}$ Six different concentrations of NAD(P)H between 50 and $300 \mu \mathrm{M}$ were used and xylose concentration was $400 \mathrm{mM}$.

${ }^{\mathrm{c}}$ Double mutants E223A/S271A (AA) and E223D/S271A (DA)

${ }^{\mathrm{d}} \mathrm{ND}$ : Not detected 

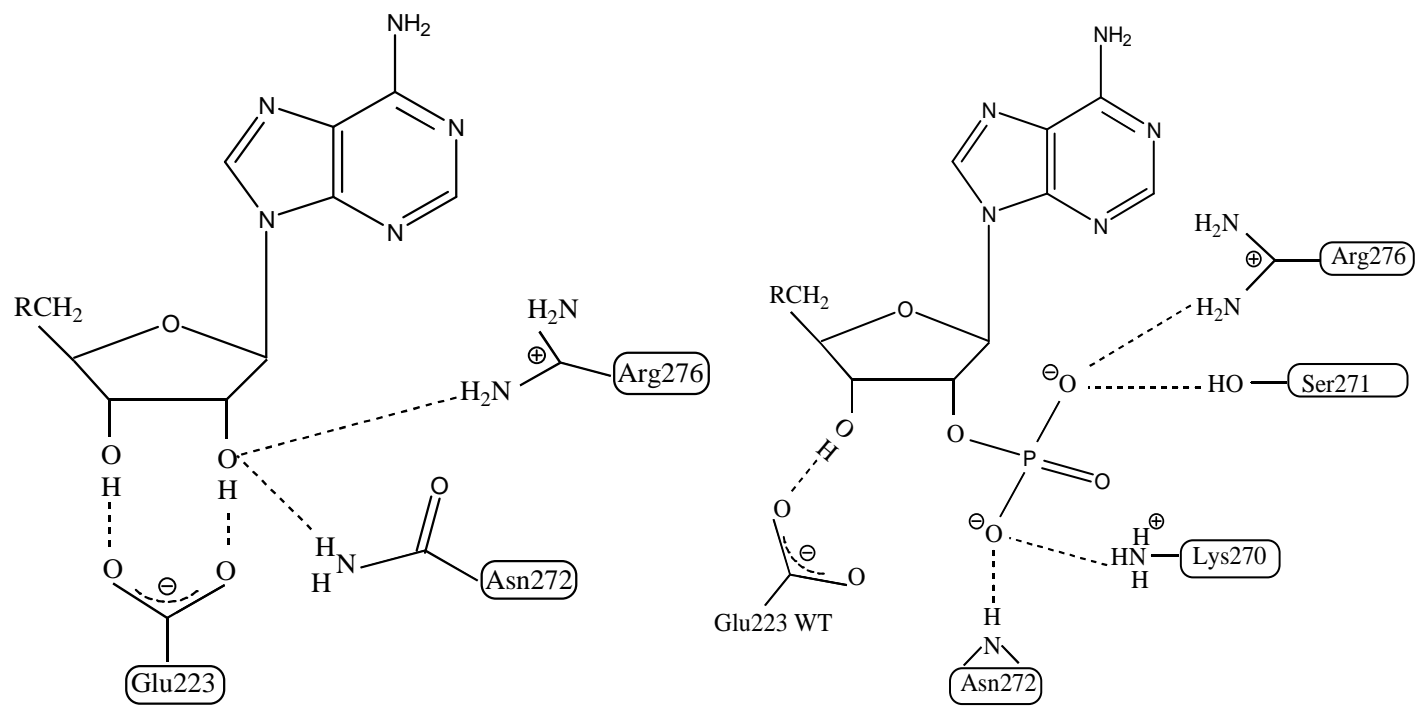

Fig. 1. Schematic diagrams showing the predicted interactions of wild-type PsXR; Lefthand panel: adenosine 2'- and 3'- hydroxy groups in the complex with $\mathrm{NAD}^{+}$and Righthand panel: adenosine 2'- and 3'- hydroxy groups in the complex with $\mathrm{NADP}^{+}$based on the coenzyme binding sites in CtXR [10].

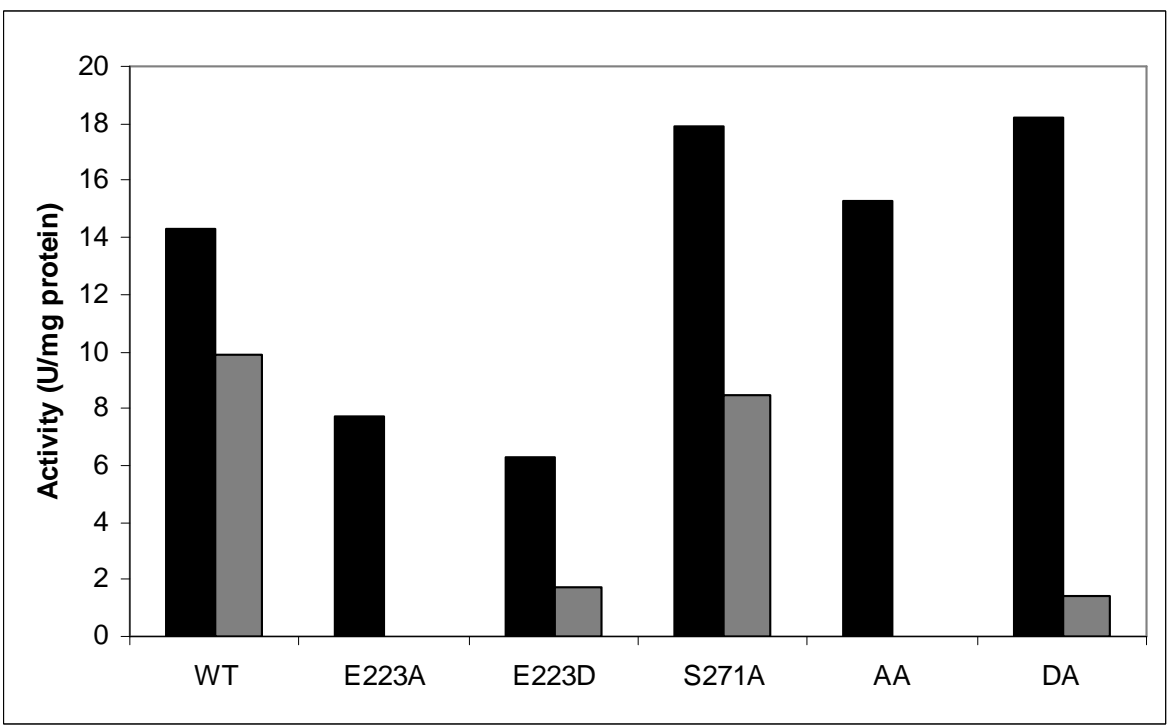

Fig. 2. Enzyme activities of PsXR wild-type and mutated enzymes. Black and grey bars indicated activities with NADPH and NADH respectively; Values are average $\pm S D, n=3$. 